ISSN 0001-6012/2019/61/4/172-176 Acta Médica Costarricense, (C) 2019 Colegio de Médicos y Cirujanos de Costa Rica

\title{
Original
}

\section{Crecimiento postraumático en pacientes sobrevivientes de cáncer infantil}

\section{(Posttraumatic growth in pediatric oncologic patients)}

\author{
Ana Yéssika Gamboa-Chaves ${ }^{1}$ y Mónica Quirós-Mata²
}

\section{Resumen}

Objetivo: estudiar el crecimiento postraumático en sobrevivientes pediátricos de linfoma o tumores sólidos, tratados en el Servicio de Oncología del Hospital Nacional de Niños, de enero de 1990 a diciembre 2013.

Métodos: este es un estudio descriptivo con pacientes sobrevivientes de cáncer quienes se encontraban en remisión, por un tiempo mayor o igual a 5 años. Se analizó las características clínicas de los pacientes (sexo, lugar de residencia, edad, tipo y localización del tumor, tratamiento recibido, presencia de metástasis o recaída), así como se efectuó una entrevista sobre la condición psicosocial de los sobrevivientes, con énfasis en las consecuencias de la enfermedad en su vida actual.

Resultados: se analizaron las características clínicas de 30 pacientes sobrevivientes. Se encontró que el tipo de tumor más común en el grupo entrevistado fue el linfoma no Hodgkin (30 \%), seguido de sarcomas (20\%). Las localizaciones tumorales mayormente observadas en estos pacientes fueron en cabeza y cuello (46,7 \%), seguidas por abdomen y pelvis $(26,7 \%)$. El tratamiento más frecuente fue la combinación de quimioterapia, radioterapia y cirugía $(37,7 \%)$. Pero cabe resaltar que el $80 \%$ de los pacientes tratados recibió quimioterapia como parte de su tratamiento y la mayoría reportó efectos adversos y dificultades relacionadas. El procedimiento quirúrgico más empleado fue la biopsia (53,3 \%). La mayoría de los pacientes no presentó recaídas (83,3 \%) ni metástasis $(93,3$ $\%)$. Con respecto a su condición actual, la edad promedio al momento de la entrevista fue de 15,9 años, y la mayoría residía en la provincia de San José (40 \%), con sus padres y hermanos (36,7 \%). La mayoría señaló reacciones positivas ante el diagnóstico, con actitudes buenas y cooperadoras. El mayor vínculo socioafectivo descrito corresponde a la familia y los amigos; un grupo importante de los pacientes recalca el apoyo institucional.

Conclusiones: a pesar de que con este trabajo no se pueden realizar generalizaciones sobre los pacientes en remisión de cáncer infantil, la información obtenida permite conocer de manera indirecta el impacto psicosocial de la enfermedad oncológica en la condición actual de un grupo de pacientes, y resalta una actitud positiva en los sobrevivientes.

Descriptores: cáncer infantil, tumores sólidos, características clínicas, resiliencia, crecimiento posttraumático, Costa Rica.

Trabajo realizado en Hospital Nacional de Niños "Dr. Carlos Sáenz Herrera". Caja Costarricense de Seguro Social.

Afiliación de los autores: 'Hospital Nacional de Niños "Dr. Carlos Sáenz Herrera". "Hospital "Dr. Rafael Ángel Calderón Guardia". Caja Costarricense de Seguro Social.

Conflicto de intereses: Declaramos que no hay conflicto de intereses.

凶moquiros@gmail.com

\section{Abstract}

Objective: to study resilience (post-traumatic growth) in pediatric cancer survivors who were treated in the Oncology Service of the National Children's Hospital, from January 1990 to December 2013.

Methods: descriptive study with cancer survivors who were in remission, for a time greater than or equal to 5 years. The clinical characteristics of the patients (sex, place of residence, age, type and location of the tumor, treatment received, presence of metastasis or relapse) were analyzed, as well 


\section{Crecimiento postraumático / Gamboa-Chaves y Quirós-Mata}

as an interview on the psychosocial condition of the survivors, with emphasis on the consequences of the disease in their current life.

Results: 30 survivors of childhood cancer were interviewed and the clinical characteristics were analized. Among the results, the most common type o tumor found was non Hodgkin lymphoma (30\%), followed by sarcomas (20\%). The tumor locations mostly observed in these patients were in the head and neck (46.7\%), followed by the abdomen and pelvis (26.7\%). The most frequent treatment was the combination of chemotherapy, radiotherapy and surgery (37.7\%). But it should be noted that $80 \%$ of treated patients received chemotherapy as part of their treatment and the majority reported adverse effects and related difficulties. The most widely used surgical procedure was biopsy (53.3\%). The majority of patients had no relapses (83.3\%) or metastases (93.3\%). Regarding their current condition, the average age at the time of the interview was 15.9 years, and the majority resided in the province of San José (40\%), with their parents and siblings (36.7\%). The majority indicated positive reactions to the diagnosis, with good and cooperative attitudes. The greatest socio-affective link described during their disease corresponds to family and friends and hospital health workers.

Conclusions: Although, with this work, generalizations cannot be made about patients in remission of childhood cancer, the information obtained allows us to indirectly know the psychosocial impact of the oncological disease in the current condition of a group of patients in our country, and highlights a positive attitude in survivors.

Keywords: childhood cancer, solid tumors, clinical characteristics, resiliencia, posttraumatico growth, Costa Rica.

Fecha recibido: 22 de octubre 2019

Fecha aprobado: 19 de septiembre 2019

"Lo que no te mata, te hace más fuerte": una expresión modificada de la original de Frederich Nietzsche, dura, pero cierta. ${ }^{1}$ La resiliencia es un proceso dinámico con el que la persona adquiere fortalezas al afrontar la adversidad. Es la capacidad de resistir, de encarar, de recuperarse y surgir de cara a experiencias difíceles de la vida. ${ }^{2}$

Un evento traumático es aquel que involucra una amenaza de muerte, o a la integridad psicológica, o una herida de gravedad, y que logra desencadenar una reacción intensa de miedo, desesperación y horror. ${ }^{3}$ En palabras de Janoff-Bulman y Frieze: "Cuando la tragedia ataca, nuestras suposiciones fundamentales son destrozadas, nuestras ilusiones protectoras son destruidas y los sobrevivientes están forzados a confrontar su propia vulnerabilidad y fragilidad." ${ }^{4}$

Se ha descrito que todos los seres humanos tenemos capacidad de resiliencia, no obstante, esta puede modificarse según la presencia o ausencia de ciertos factores, los cuales se han pretendido demostrar con estudios en niños con cáncer y sus familiares. Además, se han propuesto hipótesis que expliquen las razones que caracterizarían la capacidad de resiliencia de una persona. Por ejemplo, según el estudio de Rosenberg et al., la resiliencia proviene de 3 razones fundamentales: un rasgo prexistente que permite el crecimiento personal ante la adversidad, un proceso dinámico de adaptación positiva, un resultado final o un estado de relativo positivismo tras un evento adverso. ${ }^{5}$

Sin embargo, en ninguno de los estudios son claras las razones para comprender la capacidad de resiliencia, y más bien se sugiere que esta surge de un conjunto de factores. Por lo tanto, el entendimiento de la resiliencia y su promoción en pacientes, aún no se ha comprendido completamente, pero los beneficios alrededor de su presencia sí han sido bien descritos. ${ }^{5}$

Es importante conocer que en los pacientes que sufren una noticia traumática o amenazante para su vida, puede desencadenarse un crecimiento posterior, en el cual el personal de salud puede influir. Por ejemplo: brindar espacios para conversar y resolver dudas, mantener una comunicación clara con los familiares y los pacientes, fomentar espacios sociales, grupos de apoyo y utilizar terapias complementarias (musicoterapia, arte terapia, relajación, meditación), permiten un mejor control del dolor, de la salud mental y autoestima. ${ }^{6}$

Tener cáncer, recibir sus tratamientos prolongados, afrontar las incertidumbres de los resultados de la terapia, o saber si se va a sobrevivir, tienen sin duda un profundo efecto en el ajuste psicológico de los pacientes y, consecuentemente, un impacto en su vida. ${ }^{7}$ En sobrevivientes de cáncer se han observado síntomas relacionados con el estrés de padecer la enfermedad, el cual puede conducir a un síndrome postraumático. En cuanto a estos síntomas hay reportes de disminución en la adherencia a regímenes médicos, y aún de mayor impacto, disminución en la calidad de vida de los pacientes. ${ }^{7,9}$ Pero, con un cambio en el significado de la experiencia y un reajuste de los esquemas personales preconcebidos, estos síntomas ya no se traducen en estrés postraumático (EPT), sino más bien en un crecimiento postraumático (CPT), y en estos casos se observa un incremento del optimismo de los pacientes y en la satisfacción de su vida, así como una mejoría en las habilidades y recursos sociales, personales, y en los mecanismos de adaptación. ${ }^{9}$ 
El crecimiento postraumático fue descrito en psicología en los años noventa, cuando se observó cómo, tras ciertas experiencias traumáticas o amenazantes de vida, se podían generar cambios y beneficios para quienes las sobrellevaban. Dicho crecimiento se alcanza tras una modificación de las visiones personales, lo que permite una reconstrucción del significado del evento y, finalmente, un entendimiento de la situación. ${ }^{11}$

Encontrar el significado de situaciones como el diagnóstico de cáncer requiere un proceso. El camino a la aceptación del diagnóstico en principio se describe cargado de negatividad e incertidumbre ("¿por qué yo?, ¿por qué mi hijo, ¿qué hice mal?, ¿es esto un castigo?), documentadas en niños pequeños y adolescentes. Pero, si el proceso se acompaña de apoyo (emocional y social) y hay una suficiente reestructuración de las creencias preexistentes, se logra encontrar un significado nuevo, a partir de alguna de estas formas principales: encontrar un significado religioso ("Dios tiene el control y tiene planes para mí"), hallar beneficios secundarios a la enfermedad ("nuevas amistades o un crecimiento que de otra forma no se hubiera logrado"), o interiorizar que se es un ser especial en un contexto no religioso. ${ }^{11}$

En los pacientes sobrevivientes de cáncer en quienes se ha logrado identificar este crecimiento postraumático, se describen también cambios y beneficios como: modificaciones en sus valores y prioridades, creándose mayor conciencia por la fragilidad de la vida y la salud; variabilidad al escoger amistades o parejas, en tanto se deriva mayor satisfacción de las contribuciones emocionales de los demás; mayor autoconsciencia, pues son capaces de verse a sí mismos como personas más fuertes y orgullosas de la situación que sobrellevaron; cercanía o unión familiar, en virtud de que debido a la enfermedad, los padres suelen acercarse más a sus hijos y ser más abiertos con respecto a los sentimientos y emociones, y el surgimiento de deseos de devolver a la sociedad, dado que como parte del agradecimiento y reconocimiento de la labor de otros, en muchos pacientes nace el deseo de colaborar con otros en situaciones similares, o buscar profesiones afines (educadores, enfermeros, fisioterapeutas). $.8,8,9,11,15$

Los predictores para el crecimiento o estrés postraumático aún no están del todo claros y hay pocos estudios al respecto. Se reporta haber encontrado mayor crecimiento postraumático cuando el diagnóstico de cáncer ocurre a edades más tempranas; sin embargo, otros estudios reportan también mayor crecimiento con mayor edad. ${ }^{3,8}$ Además, el tipo de apoyo que reciben estos pacientes modifica su proceso emocional, lo que conlleva una mejor experiencia y crecimiento cuando hay un mayor grupo de soporte emocional, conversacional y social. ${ }^{3}$

Por ende, la resiliencia y el crecimiento postraumático en pacientes con cáncer y sobrevivientes de cáncer, traen beneficios personales. Hasta el momento, la mayoría de las investigaciones se han enfocado en obtener información clínica de los pacientes o la medición de la respuesta terapéutica; esta investigación pretendió conocer no solo algunas características clínicas de los pacientes analizados, sino también su condición psicosocial actual tras sobrevivir al diagnóstico de cáncer infantil. Por 10 tanto, el objetivo de este trabajo fue estudiar la resiliencia en sobrevivientes pediátricos de llinfoma o tumores sólidos tratados en el Servicio de Oncología del Hospital Nacional de Niños, de enero de 1990 a diciembre de 2013. No se hace énfasis en la parte fisica donde la bibliografía cientifica es exhaustiva sobre las secuelas de los tratamientos.

\section{Métodos}

El estudio es descriptivo y se basa en la información clínica y psicosocial obtenida de una entrevista semiestructurada, realizada a pacientes sobrevivientes de cáncer infantil, con un periodo de remisión mayor o igual a 5 años.

El estudio fue aprobado en la sesión número SABI/CLOBI 003-2016, por el Comité Local de Bioética e Investigación.

Se incluyeron pacientes sobrevivientes de cáncer con el diagnóstico de linfoma o tumor sólido, tratados en el Servicio de Oncología del Hospital Nacional de Niños, desde 1990 hasta diciembre de 2013, con 5 o más años de remisión clínica. Se excluyeron pacientes que no quisieron participar de la entrevista, pacientes quienes no completaron más del $90 \%$ de la entrevista y pacientes que se rehusaron a firmar el consentimiento informado. Este último fue firmado por los sobrevivientes mayores de edad o, en su defecto, por sus tutores legales.

De todos los pacientes se obtuvo la siguiente información: sexo, lugar de residencia, edad, tipo y localización del tumor, tratamiento recibido, presencia de metástasis o recaída, así como la condición psicosocial con énfasis en las consecuencias de la enfermedad sobre su vida actual (secuelas del tratamiento, principalmente). Se utilizaron como técnicas estadísticas para el análisis de la información la distribución de frecuencia: cruce de variables entre el sexo, la edad, el estadio, y protocolo terapéutico utilizado.

\section{Resultados}

De los 30 pacientes analizados, 14 eran mujeres (46,7 \%) y 16 hombres (53,3\%). La mayoría de los entrevistados residía en las provincias de San José (40 \%) y Cartago (20 \%); el resto se distribuyó en las demás provincias, excepto Limón, de donde no se entrevistó a ningún sobreviviente. La edad promedio encontrada fue de 15,9 años (DE: 3,6). El tiempo de traslado promedio para la cita de la entrevista fue de 2.3 horas (Cuadro 1).

Los principales antecedentes diagnósticos en estos pacientes fueron: linfoma no Hodgkin (30\%), sarcomas (20\%), linfomas de Hodgkin $(16,7 \%)$ y tumores del sistema nervioso central $(13,3 \%)$. La localización tumoral más frecuente fue a nivel de cabeza y cuello (46,7 \%), seguida de abdomen y pelvis $(26,7 \%)$ (Cuadro 1).

La mayoría de los pacientes recibió como tratamiento de primera línea una combinación de quimioterapia, cirugía y 


\section{Crecimiento postraumático / Gamboa-Chaves y Quirós-Mata}

radioterapia (37,7\%), pero en el $80 \%$ de los casos, ya fuera de forma aislada o en combinaciones, los pacientes recibieron quimioterapia. Únicamente, el 16,6 \% de los pacientes requirió una segunda línea de quimioterapia y tuvo recaídas. Uno de los pacientes con recaída se sometió a trasplante de médula ósea.

El tipo de cirugía realizado en ellos fue la biopsia (53,3 \%), seguida de la resección tumoral (43,3\%). De los pacientes a quienes se les practicó resección, dos sufrieron enucleación (uno de ellos bilateral) y uno amputación de la extremidad superior izquierda.

E1 100\% de los pacientes estaba soltero y vivía en su mayoría con ambos padres y con hermanos (36,7\%). Con respecto a la escolaridad, la mayoría cursaba secundaria (60\%). Únicamente tres sobrevivientes contaban con títulos universitarios y laboraban (10\%).

Cuando se les interrogó sobre la reacción inicial al diagnóstico, la mayoría refirió haberse mostrado cooperadora y con una buena respuesta (50\%), no obstante, un porcentaje no despreciable refirió síntomas más negativos: enojo, depresión y miedo (20\%). Siete pacientes no recordaron su reacción $(23,3 \%)$ y los restantes $(6,7 \%)$ reportaron una mezcla de emociones (enojo, miedo, cooperación, depresión). Con respecto a la

\section{Cuadro 1. Características clínicas de los pacientes sobrevivientes de cáncer infantil, Servicio de Oncología del Hospital Nacional de Niños, 1990 - 2013}

\begin{tabular}{|lc|}
\hline Característica & Número (\%) \\
\hline Sexo & \\
Femenino & $14(46,7)$ \\
Masculino & $16(53,3)$ \\
Provincia de residencia & \\
San José & $12(40)$ \\
Cartago & $6(20)$ \\
Alajuela & $4(13,3)$ \\
Heredia & $3(10)$ \\
Puntarenas & $3(10)$ \\
Guanacaste & $2(6,7)$ \\
Limón & $0(0)$ \\
Edad y grupo etario & \\
Promedio de los sobrevivientes & \\
12 a < 15 años & 15,9 años \\
15 a < 18 años & $7(23,3)$ \\
Mayor a 18 años & $19(63,3)$ \\
Diagnósticos oncológicos & $4(13,3)$ \\
Linfoma no Hodgkin & \\
Sarcoma & \\
Linfoma Hodgkin & $2(6,7)$ \\
Tumores del sistema nervioso central & $2(6,7)$ \\
Retinoblastoma & $1(3,3)$ \\
Tumor de Wilms & $1(3,3)$ \\
Carcinoma nasofaríngeo & $4(16,7)$ \\
Neuroblastoma & \\
& \\
& \\
\hline
\end{tabular}

reacción de sus familiares, el 40 \% reportó buena respuesta y cooperación. Un 20 \% de los familiares reaccionó con emociones más negativas y otro $40 \%$ con sentimientos varios (enojo, miedo, depresión, cooperación. Se consultó también sobre la respuesta de los amigos cercanos, y la mayoría asoció emociones positivas (56,7 \%). Un 23,3 \% reportó distanciamiento, trato diferenciado o respuesta negativa. El resto no especificó o refirió tener corta edad al momento de su diagnóstico.

Sobre el apoyo recibido durante el evento de su enfermedad, la mayoría recalcó el apoyo familiar (20 \%) e institucional (30 $\%)$, como su soporte más importante.

Sobre las dificultades durante la evolución de su enfermedad, la mayoría reportó adversidades secundarias al tratamiento con quimioterapia (53,3\%) y al dolor (16,7 \%). Dichosamente, la mayoría no asoció dificultades actuales (40 \%); solo el 16,7 \% de los pacientes reportó problemas con su salud actual y el 23,3 \% refiere dificultades varias (académicas, emocionales y sociales).

Entre las consecuencias positivas, el 93,3 \% señaló fortalezas emocionales, sociales y académicas. Y la mayoría le otorgó mucho más valor a su familia y salud $(56,6 \%)$.

\section{Discusión}

En la mayoría de los casos analizados se requirió quimioterapia para su manejo, aunque se documenta que un grupo importante requirió del uso de triple terapia (quimioterapia, radioterapia y cirugía), resaltándose cómo estos pacientes fueron sometidos a tratamientos más intensos y con mayores complicaciones. Esto adquiere relevancia en el estudio, ya que se encuentran en la bibliografía descripciones sobre las preocupaciones de los sobrevivientes con cáncer en su reincorporación a la sociedad (capacidad de desarrollar relaciones afectivas, tener hijos, contraer matrimonio, culminar estudios o laborar).2,13

De los pacientes que sufrieron recaídas y requirieron una segunda línea de tratamiento, solo una fue sometida al proceso de trasplante de médula ósea y en la actualidad se desenvuelve adecuadamente en la sociedad y es madre de un niño. Vale la pena resaltar que entre la bibliografía, estos pacientes trasplantados representan un grupo particular; ya que se consideran de alto riesgo para su reincorporación a la sociedad tras el tratamiento recibido. En ellos se han realizado evaluaciones sobre el desarrollo verbal, cognitivo, facultades para lectura y comprensión matemática, documentándose un buen rendimiento en la mayoría. ${ }^{14}$

En los pacientes a quienes se les realizó resección tumoral, se encontraron dos casos donde lo que se hizo fue enucleación (una de ellas bilateral), y otro de amputación del miembro superior izquierdo. En la actualidad reportan complicaciones relacionadas con su salud (adaptación a ser no vidente y dolor neuropático), aunque afirman mayores beneficios y consecuencias positivas secundarias a la enfermedad afrontada. En la bibliografía se describe cómo en este grupo de pacientes 
hay riesgos particulares (efectos a largo plazo en su funcionalidad física y necesidad de reajuste para reincorporación a la sociedad), pero cursan con un desarrollo satisfactorio de relaciones con sus pares y de su mundo, partiendo de una nueva imagen de sí mismos. Incluso hay reportes de menos síntomas depresivos y mejor vida sexual. También estos pacientes se sienten orgullosos de sí mismos por haber sobrellevado la batalla del cáncer y lucen con orgullo su muñón, cicatriz o prótesis, con lo que demuestran ser vencedores. ${ }^{2,15}$

Con respecto a la condición actual de los sobrevivientes, su estado civil y escolaridad se asocia principalmente con su edad adolescente.

Al abordar las reacciones vividas en el momento del diagnóstico por los sobrevivientes, sus padres y amigos, predomina una actitud positiva y cooperadora en los tres grupos. El enfoque que se le atribuye a la noticia y el apoyo emocional y social durante el proceso de enfermedad son desencadenantes de crecimiento o estrés postraumático, y estos últimos se asocian o disocian de beneficios generales para los sobrevivientes. ${ }^{2,3,8,9}$ Todas estas fortalezas conllevan un efecto positivo sobre relaciones sociales, conceptos existenciales, bienestar psicológico y espiritual, y permiten un mayor control del estrés. ${ }^{11,16}$

El apoyo reconocido por los pacientes en este análisis se otorga a la familia y al equipo de salud institucional. Es preciso recalcar que los recursos con los que cuentan el paciente y la familia, representan un importante factor protector. 716

Es claro que durante el tratamiento y la evolución de la enfermedad, los pacientes pueden sufrir múltiples complicaciones asociadas a los tratamientos. Se documentó que la mayoría refirió efectos adversos a la quimioterapia o dolor. Estas complicaciones se asocian con la intensidad de los tratamientos, pero a su vez son síntomas esperados en el contexto del cáncer infantil. Sin embargo, a pesar de haber manifestado tales complicaciones, en la actualidad es una minoría la que describe dificultades.

A pesar del esfuerzo que conlleva fomentar y participar en estas tareas, son mayores los beneficios que se producen en la calidad de vida de los pacientes, y conviene iniciar y divulgar programas institucionales que busquen este bienestar. En palabras del profesor Massera: "No debemos considerar que gran parte de los curados sufran estrés postraumático sino explorar, conocer y reforzar el concepto de que la mayoría reflejan un crecimiento postraumático". ${ }^{10}$

En este trabajo no se pudo explorar más que variables básicas sobre la condición actual, debido a que no se contaba con un tiempo amplio para las entrevistas. Pero se logra marcar un precedente en tanto la bibliografía insiste abundantemente en las secuelas negativas de los tratamientos. En este caso se intenta resaltar las fortalezas y contribuir con datos propios, a desmitificar el concepto de cáncer infantil. Además, es una oportunidad para que la persona tome consciencia del valor de su atención empática y reconozca el esfuerzo de sus familias y las instituciones para ayudarlos.

\section{Referencias}

1. Evencio Flores Gutiérrez. Lo que no te mata, te fortalece. Recuperado el 8 de octubre de 2018. En : http://www.cronica.com.mx/notas/2014/858497. html

2. Teall T, Barrera M, Barr R, Silva M and Greemberg M. Psychological resilience in adolescent and young adult survivors of lower extremity bone tumors. Pediatr Blood and Cancer. 2013; 60: 1223-1230

3. Jim S.L.H. Jacobsen P.B. Posttraumatic Stress and Posttraumatic Growth in Cancer Survivorship: A Review. Cancer J 2008;14: 414-419

4. Janoff-Bulman R. Frieze I. A Theoretical Perspective for Understanding Reactions to Victimization. J Soc Issues. 1983;39: 1-17

5. Rosenberg A. Baker S. Syrjala K,L. Back A.L. Wolfe J. Promoting Resilience among Parents and Caregivers of Children with Cancer. J. Palliat Med. 2013; 16:645-652

6. Kelly K, Hooke M, Ruccione K, Landier W, Haase J. Children's Oncology Group nursing research framework. Semin Oncol Nurs. 2014;30:17-25

7. Woodgate R. A review of the literature on resilience in the adolescent with cancer: part II. J Pediatr Oncol Nurs. 1999; 16: 78-89

8. Docherty S. Rob S. Phillips-Salimi C. Cherven B. Stegenga K. HendricksFerguson $\mathrm{V}$ et al. Parental perspectives on a behavioral health music intervention for adolescent/young adult resilience during cancer treatment: report from the Children's Oncology Group. J Adolesc Health. 2013;52: 170178

9. Tillery R. Howard Sharp K. Okado Y. Long A. Phipps S. Profiles of Resilience and Growth in Youth With Cancer and Healthy Comparisons. J Pediatr Psycholy, 2016; 41:290-297

10. Massera G, Chesler M, Zebrack B, Dangio JC. Cure is not enough: One slogan, two paradigms for pediatric Oncology. Pediatr Blood Cancer. 2013;60:10691070

11. Duran B. Posttraumatic growth as experienced by childhood cancer survivors and their families: a narrative synthesis of qualitative and quantitative research. J Pediatr Oncol Nurs 2013: 30: 179-197

12. Scheurer M. Lupo P. Bondy M. Epidemioloy of Childhood Cancer. En: Pizzo P. Poplack D. Principles and Practice of Pediatric Oncology. Sétima edición. 2016

13. Thompson AL, Marsland A, Marshal P, Tersak JM. Romantic relationships of emerging adult survivors of childhood cáncer. Psycho-Oncology. 2009;18:767774

14. Carpentieri SC, Diller LR. Neuropsychological resiliency after treatment for advanced stage neuroblastoma. Bone Marrow Transplant. 2005; 35:11171122

15. Phipps S, Peasant C, Barrera M, Alderfer M, Huan O, Bannatta K. Resilience in children undergoing stem cell transplantation: results of a complementary intervention trial. Pedaitrics. 2012;129:e762-70

16. Greeff A, Vansteenwegen A and Geldhof A. Resilience in families with a child with cáncer. Pediatr Hematol Oncol. 2014;31:670-679 